\title{
Microcrédito y pobreza. La experiencia del programa Microempresas Sociales de Banmujer en Chiapas
}

\section{Microcredit and poverty. The experience of banmujer's social microenterprise program in Chiapas}

\author{
Emma Del Carmen Aguilar-Pinto* \\ Esperanza Tuñón-Pablos* \\ Federico Morales-Barragán**
}

\begin{abstract}
This article analyzes the experiences of beneficiaries of Banmujer's Social Microenterprises program which has been in operation in Chiapas since 2001, based on the conceptual trilogy of the poor (subject), poverty (context), and impoverishment (process) as proposed by Vega-Estrada. Our results show that the role of microcredit in poverty eradication must not be overestimated and that while they can contribute to reverse the process of impoverishment of poor people, do not affect the structural problem of poverty. With this we hope to contribute to the discussion about whether women microcredit is an effective tool to combat poverty.
\end{abstract}

Keywords: Microcredit, poverty, impoverishment, women, Chiapas.

\section{Resumen}

Se analiza la experiencia de beneficiarias del programa Microempresas Sociales (MES) que opera en Chiapas desde 2001, a partir de los conceptos de pobre (sujeto), pobreza (contexto) y empobrecimiento (proceso), propuestos por VegaEstrada. Los resultados muestran que el papel de los microcréditos en la erradicación de la pobreza se ha sobrevalorado y que, si bien pueden contribuir a revertir el proceso de empobrecimiento, no inciden en el problema estructural de la pobreza. Con esto esperamos contribuir a la discusión en torno a si el microcrédito femenino es una herramienta efectiva de combate a la pobreza.

Palabras clave: microcrédito, pobreza, empobrecimiento, mujeres, Chiapas.

*El Colegio de la Frontera Sur, correo-e: emma.aguilar.p@gmail.com, etunon@ecosur.mx

**Universidad Nacional Autónoma de México, correo-e: jfmoralesb@gmail.com 


\section{Introducción}

Emplear los microcréditos como estrategia para aliviar la pobreza en regiones vulnerables tuvo un gran impulso tras la creación de la institución holandesa Oikocredit, en 1975, y del Grameen Bank, en 1976, en Bangladesh. A fines de la década de los noventa, al realizarse por primera vez la Cumbre Global del Microcrédito (durante 1997 en Washington), se estableció el objetivo de atender a 100 millones de los hogares más pobres del mundo para crear autoempleo y contribuir al complimiento de los Objetivos de Desarrollo del Milenio. En este marco, los gobiernos debieron generar estrategias de promoción de microcréditos para atender a la población marginada, vulnerable y excluida del sector financiero tradicional.

En 2004, la Organización de Naciones Unidas (ONU) declaró el 2005 como el Año Internacional del Microcrédito, con el objetivo de promoverlo como una estrategia contra la pobreza. La ONU instó a los gobiernos a generar programas de microcrédito para disminuir la pobreza, fomentar el trabajo por cuenta propia y generar ingresos.

Al recurrir al enfoque propuesto por Vega-Estrada (2009, 2012 y 2014), que permite distinguir entre el sujeto (pobre), contexto (pobreza) y el proceso (empobrecimiento), podemos entender que, si bien la iniciativa de fomentar trabajo por cuenta propia -de forma consistente y sostenida- puede revertir la situación de empobrecimiento, no incide en el problema estructural de la pobreza que no puede disminuirse en el mediano plazo.

El objetivo de este artículo es analizar el papel de un programa de microcrédito enfocado a la creación de microempresas sociales para mejorar el ingreso de mujeres en situación de pobreza, mediante la asignación de microfinanciamientos y como parte de una política estatal transexenal. Realizamos esta valoración a partir del estudio de los mecanismos institucionales en los que se desarrolla el programa y de las experiencias personales de las beneficiarias en torno a los efectos del mismo en su condición de pobreza, generación de empleo, mejoramiento de su ingreso familiar y a través de la capacitación que se les proporcionó en las primeras etapas del programa. Con los resultados de nuestra investigación buscamos aportar a la discusión en torno al microcrédito femenino y su contribución en el combate a la pobreza. 


\section{Antecedentes}

\subsection{La política de microcréditos en México}

De acuerdo con el Consejo Nacional de Evaluación de la Política de Desarrollo Social (Coneval, 2009), las políticas públicas de combate a la pobreza en México que impulsan la actividad financiera de ahorro y crédito para el sector de la población, excluido del sector financiero tradicional, se realizan mediante siete programas federales: Programa de Apoyo al Sector Turismo, Programa de Apoyo para Acceder al Sistema Financiero Rural (Paasfir), Fondo Nacional para Empresas en Solidaridad -hoy Instituto Nacional de la Economía Social (Fonaes-Inaes)- Fondo de Apoyo para la Micro, Pequeńa y Mediana Empresa (Fondo PyME), Programa Nacional de Financiamiento al Microempresario (Pronafin), Proyecto Regional de Asistencia Técnica al Microfinanciamiento Rural (Patmir) y Fondo de Financiamiento a Mujeres Rurales (Fommur). Cabe señalar que este último es el único programa federal de microcréditos destinado exclusivamente a la población femenina y rural del país.

Al decir de Alpízar y González-Vega (2006), en México la implementación de esta política de microcréditos basada en el modelo de microfinanzas rurales ha reemplazado paulatinamente al enfoque financiero formal. Esto se puede observar en la creación en 2003 de la Financiera Rural (Finrural), y en 2012 del Banco Nacional del Ahorro y de Servicios Financieros (Bansefi), instituciones que sustituyeron a dos entidades importantes, Bonos del Ahorro Nacional (Pahnal) y el Banco de Desarrollo Rural (Banrural), respectivamente.

El impacto de esta política federal de microcréditos ha sido analizada por diversas investigaciones (Arellano-Gault et al., 2006; Bustos-Villegas, 2010), así como las iniciativas estatales y municipales de programas de microfinanzas (Varela-Zuñiga, 2007; Tuñón-Pablos et al., 2007; GonzálezMartínez y Basaldúa-Hernández, 2009; Sampayo-Paredes y HernándezRomero, 2009; Millán-Valenzuela, 2009; Medina-Núñez y Florido-Alejo, 2010). Otros autores evalúan a las entidades no gubernamentales y a otras iniciativas que promueven el microcrédito (Cotler-Ávalos y RodríguezOreggia, 2010; Esquivel-Martínez, 2010; Vázquez-Jiménez, 2012; Delalande y Paquette, 2007) y otros más evalúan el impacto de los microcréditos que otorga una Institución de Asistencia Privada (IAP), el Centro de Apoyo al Microempresario (CAME) (Zapata-Martelo et al., 2004a; 2004b; 2004c), así como la relación entre microcréditos y pobreza femenina (García-Pinar, 2012; García-Horta et al., 2014). 


\subsection{El Programa Microempresas Sociales: un esbozo}

En Chiapas, por iniciativa estatal y en armonía con la política nacional de microcréditos, durante 2001 se creó una instancia destinada a atender a mujeres pobres llamada Beneficio y Apoyo de las Mujeres (Banmujer) que fue innovadora para el contexto, primero por su intención de asignar microfinanciamientos para mujeres en situación de pobreza y, segundo, por la estrategia de capacitación que se diseñó para acompañarlas y que resultó pionera al acercar a las mujeres chiapanecas información sobre temas de género y empoderamiento vinculado a cuestiones técnico-administrativas que les ayudarían en la creación y funcionamiento de sus microempresas.

Los programas implementados fueron "Una semilla para crecer" (en lo sucesivo USPC) y Microempresas Sociales (en adelante MES) que operan a través del fideicomiso público de inversión Fondo de Apoyo a la Economía Social (FAES). En el diseño de los programas se estableció que debían ser instrumentados de manera secuencial, primero USPC y luego MES, en tanto que éste último atiende la continuidad en el uso de microcréditos de las beneficiarias de USPC que posean las condiciones necesarias para instalar una microempresa.

Ambos programas estuvieron destinados a asignar microfinanciamientos $^{1}$ accesibles a mujeres organizadas en grupos solidarios para fomentar el ahorro, generar empleos y mejorar el ingreso de las mujeres (Sedem, 2011a). Cabe señalar que durante seis años estos fueron los únicos programas estatales en su tipo.

Si bien MES al principio financió a grupos de mujeres y hombres, en 2002 -siguiendo el paradigma de las finanzas populares- focalizó su atención en la población femenina en condición de pobreza y marginación que se encuentra excluida del sector financiero tradicional y empezó a apoyar la creación de microempresas entre grupos de mujeres, mediante la dispersión de pequeños créditos. En el periodo gubernamental 20062008 se implementó otro programa estatal de microcréditos, el Fondo de Empresas Sociales, que no está exclusivamente destinado a las mujeres y que se opera a través de BanChiapas, órgano administrativo desconcentrado que depende de la Secretaria de Hacienda del Estado de Chiapas y

\footnotetext{
${ }^{1}$ El programa USPC asigna microfinanciamientos individuales para mujeres con montos de $\$ 1000, \$ 1500$ y $\$ 2000$ en tres etapas, respectivamente, para que las beneficiarias se capitalicen, ahorren, mejoren sus ingresos y sus condiciones de vida y de trabajo (Sedem, 2011a: 5). MES asigna créditos que van de $\$ 3^{\prime} 000$ hasta $\$ 10,000$ y busca impulsar la creación de Microempresas Sociales. En ambos casos, la tasa de interés global de los financiamientos es de 1.4 por ciento; deben realizarse pagos semanales y las mujeres deben organizarse en grupos solidarios en USPC de 12 a 20 mujeres y en MES de tres a cinco.
} 
que resulta muy parecido, en su operación y funcionamiento, al programa MES.

\subsection{Los referentes teóricos: pobre, pobreza y empobrecimiento}

En México, el Coneval (2012) establece que una persona es pobre cuándo tiene, por lo menos, una carencia social de los seis indicadores considerados: rezago educativo, acceso a servicios de salud, acceso a la seguridad social, calidad y espacios de la vivienda, servicios básicos en la vivienda y acceso a la alimentación; y cuándo su ingreso es insuficiente para adquirir los bienes y servicios que requiere para satisfacer sus necesidades alimentarias y no alimentarias.

Para analizar la pobreza, en este trabajo tomamos como referente los conceptos de pobre (sujeto), pobreza (contexto) y empobrecimiento (proceso) que propone Vega-Estrada (2009, 2012 y 2014), ya que su perspectiva nos revela que el enfoque tradicional de medición de la pobreza se centra en medir al pobre y deja sin atención otras manifestaciones del problema como los procesos económicos, sociales y culturales por los cuales las personas se convierten en pobres (empobrecimiento).

Para Vega-Estrada (2014) el concepto de pobre (sujeto) refiere al conjunto de personas pobres carentes de una serie de recursos mínimos para subsistir, mientras que la pobreza puede verse desde un ángulo cuantitativo y otro cualitativo. El primer aspecto se ha difundido ampliamente y se mide en función de cantidades (por ejemplo el total de personas pobres que existen en un país o región), con base en la comparación de líneas económicas ligadas a una canasta básica y alude a una situación de comparación frente a un todo. Por su parte, el ángulo cualitativo se refleja en un contexto, situación, condiciones, circunstancias y condiciones concretas de la existencia de los individuos pobres que son generados por la cultura y la historia de un país.

Sobre el empobrecimiento, el autor afirma que tiene una connotación económica y social, y que es un proceso de largo aliento que permite ver las condiciones de vida que llevaron a los individuos a tener la condición de pobres; este proceso da origen a los hogares pobres (pobreza) y a los pobres (sujetos). Vega-Estrada (2014: 87) define este concepto como un "conjunto de hechos, eventos o acciones que ocasionaron que un sector de la población resultara confinado al lugar más bajo o más incómodo de una escala de acceso económico-social a bienes y servicios".

Este proceso se manifiesta en la vida de los pobres de muchas maneras, por ejemplo, al ser despedidos de su empleo, al no acceder a un aumento salarial o al ser rescindidos de prestaciones sociales, cuando desaparecen fuentes de empleo o ante el aumento del precio de los productos básicos, 
las tasas de interés desfavorables o los impuestos desiguales. Para VegaEstrada (2014), estos aspectos no son visibles en los resultados de las evaluaciones que se centran en medir-calificar al pobre (sujeto) y no a la pobreza (contexto) o al empobrecimiento (proceso) y señala que estas categorías se combinan y juntas determinan la presencia de nuevos pobres y la persistencia de quienes ya han sido nombrados así. La perspectiva propuesta por el autor muestra que en México el programa oficial de combate a la pobreza (Prospera-Progresa-Oportunidades-Solidaridad) no ha surtido efecto debido a que ni los objetivos ni las acciones de éste inciden en la estructura y se enfocan únicamente en la atención del pobre, lo que permite entender la continuidad del mismo y los escasos resultados alcanzados.

En nuestra investigación utilizamos el concepto pobre para denominar al grupo de mujeres que reciben microfinanciamiento del programa MES; el contexto de pobreza en el que se encuentran queda plasmado en la lejanía de algunas localidades en las que viven, la escasez de medios de trasporte y otros medios de comunicación, y el empobrecimiento se expresa en el desempleo, la falta de oportunidades laborales, la necesidad de recurrir a créditos de micro financieras privadas para apuntalar sus microempresas, destinar los ingresos que se generan por la microempresa a la subsistencia familiar y la estrategia de fungir como prestanombres para que el esposo o algún familiar de sexo masculino tenga acceso al microcrédito de MES e instale o consolide una microempresa.

\section{Metodología}

Seleccionamos a beneficiarias del programa MES que viven en seis municipios atendidos por la Delegación I centro de la Secretaría para el Desarrollo y el Empoderamiento de las Mujeres, instancia que a través de la Subsecretaria de Economía Social de Banmujer, asigna los microfinanciamientos. Estos municipios son Berriozábal, Chiapa de Corzo, Cintalapa, Ocozocoautla, Villaflores y Tuxtla Gutiérrez. De las 682 mujeres atendidas por MES en estos municipios, se consideró una muestra de 158 mujeres que representan $23 \%$ del total de la población atendida y, de éstas, se seleccionaron 97 para ser entrevistadas, mismas que representan $61.4 \%$ de la muestra y $14.2 \%$ de la población atendida por el programa. Las mujeres se encuentran integradas en 45 grupos solidarios y viven en distintas localidades, ejidos, colonias y barrios de los municipios seleccionados.

El criterio fue seleccionar a la población que se encuentra en situación de pobreza y vulnerabilidad, de acuerdo con los lineamientos federales y estatales contemplados por los programas sociales que reciben y que están basados en la Línea de Bienestar establecida por el Coneval (2012), así 
como el ser beneficiarias de, al menos, dos de estos programas, incluido el MES. Asumimos así que las 97 mujeres beneficiarias de nuestro estudio han sido, al menos en dos ocasiones, identificadas como pobres en base a los requerimientos del Coneval.

De esta manera, $77.3 \%$ de nuestra muestra indicó recibir paralelamente a MES un programa federal, $13.4 \%$ uno estatal y $9.3 \%$ programas de ambas fuentes. Siete de cada 10 beneficiarias $(72.2 \%)$ que reciben programas federales reportó ser beneficiaria de Prospera; 6.2\% del programa 70 y más, pensión para adultos mayores; $2.1 \%$ recibe apoyo del Programa Alimentario Sin Hambre, y 1\% del programa de Apoyo a la Economía Social, modalidad Crece. En cuanto a los programas estatales, 7.2\% afirmó recibir el programa Amanecer; 3.1\% el programa Bienestar de Corazón a Corazón para madres solteras, y 1\% tiene apoyo del Fondo de Empresas Sociales de BanChiapas. De las que reciben programa federal y estatal, $5.3 \%$ son apoyadas por el Fondo de empresas sociales (BanChiapas) y por el Programa de Apoyo para la Mujer Emprendedora (Promete); 3.1\% cobran Amanecer y Pensión para adultos mayores, y 1\% el Programa Alimentario Sin Hambre y Bienestar de Corazón a Corazón.

A las mujeres integradas en esta muestra se les aplicó un cuestionario para recabar información sobre datos socioeconómicos, laborales, experiencia previa en el proyecto, uso del microcrédito y su percepción sobre el impacto del microcrédito en el negocio. A la par de la aplicación del cuestionario se realizaron entrevistas semiestructuradas durante julio y agosto de 2014 para conocer la opinión de las mujeres acerca del microcrédito como atenuante de la pobreza. En otro momento del levantamiento de datos se realizaron entrevistas semiestructuradas a funcionarios que estuvieron a cargo de la marcha del programa en diferentes periodos de su aplicación.

La estrategia que empleamos comprende el análisis del programa través de fuentes secundarias, la percepción de las beneficiarias y la experiencia de algunos funcionarios del programa. Para eso nos basamos en un modelo de evaluación de impacto participativa, ya que ésta enfatiza la percepción social o comunitaria de los efectos y utilidad del programa a partir de las opiniones de las y los involucrados en el mismo (Apodaca, 1999). Para su abordaje, se diseñó un estudio observacional (no experimental) de tipo transversal por tratarse de un programa de cobertura parcial $^{2}$. En términos éticos, el estudio contó con el consentimiento in-

${ }^{2}$ Rossi et al. (2004) distinguen entre los diseńos de evaluación de los programas de cobertura total y los de cobertura parcial. Los primeros son los que obligan a tomar como referencia sólo a grupos que hayan sido objeto de la intervención (no es posible la comparación entre grupos de control, se hace con el propio grupo y se denominan controles reflexivos). En cambio, para la evaluación de programas de cobertura parcial se encuentran los diseńos no experimentales o cuasi-experimentales que se adaptan a diferentes situaciones y necesidades de la evaluación y en los que 
formado de todas las mujeres entrevistadas y, por criterio de confidencialidad, todos los nombres que aparecen en el texto son ficticios.

\section{Resultados}

Para presentar los resultados de esta sección identificamos tres periodos consecutivos del programa: el primero corresponde al nacimiento de Banmujer y creación de MES en el sexenio 2000-2006, el de expansión de los programas de microfinanciamiento en el periodo gubernamental 2006-2012 y el último que inició en 2012 y se extiende a 2018 y que, para los fines de esta investigación, acotaremos en 2014. En cada uno abordaremos cómo se realizó la selección de beneficiarias y los procesos de capacitación que han implementado. Al final plantearemos las características de las microempresas y las opciones de empleo o autoempleo que las beneficiarias de MES concretan. En esta parte articulamos la información institucional y la dimensión de la experiencia de las beneficiarias, así como la de algunos funcionarios involucrados en el programa que se entrevistaron entre 2013 y 2016, para poner de manifiesto algunas inconsistencias en el diseño y las tensiones que existen en la aplicación del mismo.

\subsection{El proceso de selección de beneficiarias dentro del programa MES}

$\mathrm{Al}$ analizar las reglas de operación vigentes del programa encontramos que la población objetivo son las mujeres en situación de pobreza y que el objeto del programa es impulsar la creación de microempresas para generar empleos, mejorar el ingreso y contribuir al bienestar de los grupos solidarios de mujeres (Sedem, 2011a). Observamos que, institucionalmente, el programa carece de mecanismos para elegir a las beneficiarias -no se estipula la aplicación de un cuestionario socioeconómico u otros instrumentos que permitan detectar su condición- y esto impacta en el logro de objetivos.

La cuestión de hacia quién se dirige el programa microfinanciero depende de la misión de la institución que promueve el programa. Al decir de Conde-Bonfil (2005) esta misión puede contemplar la atención a necesidades financieras de personas muy pobres y de poblaciones vulnerables muy específicas, en ese caso es inminente asegurarse de que se llegue

usualmente se toma un grupo de control y un grupo en tratamiento. En esta investigación elegimos grupos solidarios que ya habían sido beneficiados por MES tiempo atrás (más de dos años), que ya no recibían el microcrédito (grupo control) y otros que eran de reciente ingreso (un ańo o menos) al programa (grupo en tratamiento). 
a esos grupos (enfoque hacia grupos específicos) o atender a personas que carecen de servicios financieros por lo que no es relevante centrarse en el sector de los más pobres (enfoque de exclusión/inclusión). Cada enfoque tiene su propia metodología e instrumentos para identificar a su población. Una característica importante de los programas de microcrédito es que emplean una estrategia de focalización para elegir a la población más necesitada con el menor nivel de recursos económicos, especialmente las mujeres, sin pedirles ninguna garantía para su inversión (Mejía-Flores, 2004).

$\mathrm{Al}$ respecto, las reglas de operación del programa MES establecen que: "El crédito está dirigido exclusivamente a mujeres que no tengan acceso al crédito institucional y privado, que no tengan acceso a la banca comercial y/o de desarrollo o de otras instituciones o programas gubernamentales. Con atención preferencial a aquellas que hayan cumplido de manera exitosa las fases del programa Una semilla para crecer” (Sedem, 2011a: 9). En la muestra encontramos que $79.4 \%$ de las mujeres participaron en el programa USPC, tal como lo establecen las normas, mientras que $20.6 \%$ no lo hizo y contaron directamente con el apoyo de MES, lo que deja ver una posible asignación discrecional de los créditos. Del porcentaje de beneficiarias que sí estuvo en el programa USPC ( $n=77)$, 66.2\% estuvo en los tres ciclos obligatorios que se establecen, $23.4 \%$ estuvo en más de tres ciclos por no pagar el financiamiento adecuadamente o por solicitar un nuevo crédito y $10.4 \%$ restante estuvo sólo dos ciclos, incumpliendo la norma.

La selección de beneficiarias, al decir de los funcionarios entrevistados, ocurrió de diferente manera en cada etapa del programa. Los testimonios siguientes nos muestran lo anterior en la etapa de creación, el periodo de expansión y consolidación, y en la etapa actual:

"Al crearse Banmujer, arrastrábamos programas del DIF estatal para atención a población muy vulnerable con un enfoque asistencialista" (Entrevista Alberto, 2014).

"El principal requisito era haber pasado todos los ciclos de USPC" (Entrevista Elena, 2014).

"Apoyamos a las que fueron a la plática, organizaron su grupo y tomaron los talleres de costura, bordado, velas... porque teníamos que colocar el recurso" (Entrevista Elisa, 2014).

La primera entrevista muestra las contradicciones que observamos en el programa ya que, si bien se planteó como un programa de microfinanciamiento, en la práctica existe una tendencia a operar como un programa de fomento, tal vez derivado de la experiencia de los funcionarios involucrados en su diseño y puesta en marcha. En la segunda, se observa un énfasis la prioridad que se da a las beneficiarias de USPC, quienes conta- 
ban con elementos para formalizar una microempresa; en la tercera, se expresa que el personal técnico de la Sedem podía elegir discrecionalmente a las beneficiarias priorizando "colocar el recurso" a tiempo.

Algo común en las tres etapas es la ausencia de estrategia de focalización característica de los programas de microfinanciamiento. Así, MES asigna créditos individuales sin pedir garantías a tasas muy bajas y se centra en la atención de la población femenina excluida del sector financiero tradicional. Con ello facilita el acceso a un servicio del que las mujeres carecían, pero no necesariamente incide en sus condiciones de inequidad y algunas veces hasta puede reproducirlas.

\subsection{Capacitación}

Si bien en MES el tema de la capacitación es una función clave a cargo de las coordinaciones o delegaciones regionales por la que deben: "instrumentar a través del personal técnico acciones de capacitación y formación" (Sedem, 2011a: 15), en realidad no se especifican plazos, periodos, mecanismos, estrategias ni temas. Los resultados del cuestionario aplicado muestran que siete de cada diez (71.1\%) de las mujeres beneficiarias afirmaron no haber recibido ningún tipo de capacitación durante el ciclo del proyecto y sólo $28.9 \%$ dijeron que sí. Al analizar las variables periodo y capacitación, encontramos que quienes indicaron no recibir capacitación se concentran al final del segundo periodo e inicio del tercero (ejercicio fiscal 2012 y 2013, respectivamente). De las beneficiarias capacitadas (28.9\%), 35.7\% indicaron que los temas fueron manualidades (bordado, elaboración de velas, repostería, etc.), $21.4 \%$ expresó que fue sobre aspectos técnicos, otro $21.4 \%$ sobre derechos humanos, salud reproductiva y autoestima, $14.3 \%$ sobre temas organizativos y $7.1 \%$ sobre cuestiones financieras. La duración de la capacitación fue de uno a dos días según $57.1 \%$, mientras que $42.9 \%$ expresó que fue de tres a seis horas.

Durante el primer periodo de MES la capacitación ocupó un lugar importante, en tanto que la política estatal contempló transversalizar el enfoque de equidad de género como parte de la acción pública. La estrategia empleada en Banmujer contempló ofrecer microcréditos a mujeres pobres a la par de la realización de talleres que contribuyeran al fortalecimiento de su autoestima, promoción del empoderamiento y liderazgo. En esta etapa, la Dirección de Servicios no financieros de Banmujer realizó un trabajo intenso a través del Departamento de Formación y Capacitación, que concentró esfuerzos para la capacitación de técnicos y beneficiarias. En el área de difusión se elaboraron materiales didácticos e ilustrativos accesibles a las mujeres (libros, folletos, láminas, franelógrafos, rotafolios, manuales). Observamos que en este periodo existió un énfasis 
en promover el empoderamiento, de lo que da cuenta el siguiente fragmento de entrevista:

"Muchas mujeres eran analfabetas, trabajamos con rotafolios, ilustraciones y franelógrafos e identificábamos a quienes podrían ser formadoras en sus comunidades. Los temas fueron autoestima, liderazgo, doble y triple jornada, derechos, masculinidad, resolución de conflictos" (Entrevista Sofía, 2014).

En el segundo periodo de MES, durante el sexenio gubernamental 2006-2012, la presencia femenina de estos grupos fue visible para el poder político y se recurrió a ellas para que participaran en diversos eventos públicos. En esta etapa ocurrieron cambios importantes ya que, a nivel constitucional, la legislación estatal se reformuló para crear la Sedem (2011a) que integró a Banmujer, dando como resultado la desaparición del Departamento de Formación y Capacitación, el surgimiento del programa de capacitación Mujeres Trabajando Unidas (MTU), la disminución de los recursos destinados a capacitación y su operación cada vez más esporádica; paralelamente se destinaron mayores recursos del FAES a USPC y los grupos de éste crecieron considerablemente, mientras que los de MES disminuyeron. El FAES dejó de contar con recursos federales, se masificaron los microcréditos femeninos en USPC y posteriormente a través de un nuevo programa de créditos para locatarios y pequeños comerciantes que impulsó BanChiapas.

Otro rasgo característico del periodo fue el discurso sobre la prevención de la violencia de género. Así, para conmemorar los diez años de la creación de los programas USPC y MES, se realizaron talleres sobre prevención de la violencia hacia las mujeres y niñas, violencia familiar, paternidad y maternidad afectivas, derechos sexuales y reproductivos de las mujeres, salud materna y VIH-SIDA (Sedem, 2011b). Sin duda temas importantes que pudieron tener repercusiones positivas en las mujeres, pero que resultaban carentes de relación con el fomento de las actividades económicas de las beneficiarias y el fortalecimiento de sus microempresas, tarea que debía priorizarse si MES buscara fomentar autoempleo de forma sostenida. El siguiente testimonio de una funcionaria expresa lo ocurrido en este periodo: "En 2006, el programa USPC se volvió masivo y se le quitaron recursos al programa MES. En 2007 desaparecieron los recursos para capacitación etiquetados en Banmujer" (Entrevista Cinthia, 2106).

La última etapa de MES (2012-2014) ocurre en la actual administración estatal y se caracteriza por transformaciones paulatinas en la Subsecretaria de Economía Social-Banmujer que ocasionaron ajustes en los programas y la creación de nuevos. MES es actualmente Microempresas Sociales de Corazón y el programa de capacitación MTU se convirtió en Mujeres Trabajando Unidas de Corazón. Más allá del cambio de nombres, 
destaca la carencia de recursos para capacitar a las beneficiarias. En 2014 el programa MESC proporcionó a sus beneficiarias talleres sobre: "Como formar una microempresa, promover productos y servicios, derechos de las mujeres y ahorro en la economía... y se impartieron talleres de bordado tradicional, bordado de listón, pintura en tela, repostería, gelatina, elaboración de velas, panadería y visión emprendedora" (Sedem, 2014: $8-9)$.

El fragmento de entrevista a una funcionaria expresa qué sucede en cuanto a la capacitación en este periodo: "El programa de capacitación MTU desapareció desde 2010, sólo quedaron USPC y MES, la capacitación dejó de ser importante" (Entrevista Rocío, 2013)

Observamos que los temas de capacitación de este periodo no promueven que las microempresas diversifiquen sus productos, refuerzan que las mujeres realicen los roles de género social y culturalmente asignados, asimismo, los talleres se ofrecen en las instancias de Desarrollo Integral de la Familia (DIF), lo que contribuye a disolver el impacto que pueden tener los procesos de capacitación y formación impulsados por una instancia que otorga microfinanciamientos a mujeres para crear empresas sociales.

En un programa microfinanciero el impacto del mismo está dado por el logro de objetivos que se alcanzan mediante un fuerte componente de capacitación que permita el desarrollo de capacidades. Mungaray-Lagarda y Ramírez-Urquidy (2007) sugieren que los servicios de asistencia y capacitación de los programas microfinancieros tienen un impacto positivo al fomentar microempresas como mecanismo de combate a la pobreza y estímulo al empleo. En MES observamos que suceden dos cosas: primero una desarticulación entre objetivos y estrategias de capacitación y, segundo, que el esquema de capacitación que se trató de incorporar en la primera etapa de MES se ha ido diluyendo a lo largo de estos periodos.

Los objetivos deberían alcanzarse a través de acciones y procesos de capacitación que dotaran a las beneficiarias de lo necesario para crear o consolidar sus microempresas y, con esto, se esperaba que las mujeres se auto-emplearan de forma permanente y proporcionaran fuentes de empleo que contribuyeran a revertir el empobrecimiento. Contrario a esto, MES representa el medio a través del cual el Estado atiende a una clientela importante, sin interés en que las microempresas sean sustentables, muestra de ello es el papel al que se ha relegado la capacitación.

\subsection{Microempresas sociales}


Las microempresas constituyen las fuentes de empleo o autoempleo mediante las cuales el programa MES concreta su objetivo primordial. Sin embargo, institucionalmente no hay una definición de qué se entiende por microempresa ${ }^{3}$ social $^{4}$. Antes mencionamos que MES sigue la tendencia de las finanzas populares, que propicia el desarrollo de personas con espíritu emprendedor, familias o grupos sociales mediante la creación de pequeñas empresas que se constituyan en fuentes de empleo y autoempleo para un sector pobre. Al respecto, Mungaray-Lagarda y RamírezUrquidy (2007) señalan que este tipo de microempresas se caracterizan principalmente por su debilidad financiera, nula capacidad crediticia, mínima capacitación y falta de liquidez y solvencia. En nuestro estudio encontramos que MES, cuyo objetivo principal es apoyar la instalación o ampliación de microempresas sociales (Sedem, 2011a) -al no establecer los mecanismos institucionales adecuados para consolidar las empresas con figura legal- expresa otra inconsistencia, reflejo de su tendencia a apoyar microempresas de subsistencia las cuales operan en la informalidad, con opciones de financiamiento limitadas o nulas y con pocas posibilidades de subsistir y de fomentar empleo continuo.

Consideramos que la "instalación" refiere a una primera fase de las microempresas donde son más vulnerables y requieren de acompañamiento y capacitación en temas específicos, mientras que la "ampliación" implica que han pasado un periodo crucial de sobrevivencia, se encuentran en una situación de menor vulnerabilidad y requieren otro tipo de apoyos. Sin embargo, MES no precisa a qué se refiere con cada término y los emplea como sinónimos cuando implican dos fases distintas. Esto repercute en la carencia de un esquema de capacitación, acompañamiento y seguimiento institucional adecuado para las microempresas en cada fase, lo que restringe las posibilidades del programa de incidir en el empobrecimiento de las beneficiarias, como veremos en el siguiente testimonio de una funcionaria:

"No era necesario, lo único que tenían que hacer era integrar sus grupos y estar dispuestas a trabajar de común acuerdo" (Entrevista Elena, 2014).

\footnotetext{
${ }^{3}$ El concepto refiere a un tipo de negocio cuyo tamaño e ingresos mensuales son pequeños. Para definirla Pomar-Fernández et al. (2006: 6) proponen que se trata de una "unidad económica de producción a pequeña escala, con capital social mínimo, baja inversión, pocas ventas, no más de quince empleados y donde el dueńo se involucra en todos los procesos”. Algunos rasgos característicos son que requieren poca inversión para constituirse, emplean procesos productivos y de comercialización muy sencillos y carecen de visión a largo plazo (López-Lira, 2007).

${ }^{4}$ Mungaray-Lagarda et al. (2013) proponen un concepto que puede acercarnos a la realidad de MES, se trata de las Microempresas de Base Social (MBS), que tienen un carácter precario, surgen por la necesidad de obtener recursos económicos que se emplean para la satisfacción de necesidades básicas, operan en la informalidad, tienen restricciones económicas y son susceptibles de apoyo.
} 
Sobre el uso del microcrédito, los resultados muestran que ocho de cada 10 mujeres declaró haber utilizado el microcrédito para establecer una microempresa $(77.3 \%$ ), mientras que el restante $22.7 \%$ (22 mujeres) dijo que no lo utilizó para establecer su propio negocio. De éstas, la mitad (11) lo destinaron al fortalecimiento de un negocio familiar que invariablemente ejecuta un miembro masculino de su familia (esposo, padre, hermano o hijo), cinco guardaron el dinero para cubrir eventualidades tales como enfermedades, defunciones, graduaciones y viaje; cuatro lo utilizaron para pagar deudas y dos para construir o pagar colegiaturas, lo que permite observar que actualmente no hay un seguimiento institucional para conocer el uso y destino del microcrédito.

En cuanto al tamaño de los microfinanciamientos que recibieron las beneficiarias, tenemos que el $47.5 \%$ solicitó un crédito de $\$ 10,000 ; 42.3 \%$ pidió préstamos de entre $\$ 3,000$ a $\$ 9,900$, mientras que quienes solicitaron créditos de entre $\$ 15,000$ a $\$ 20,000$ fueron $8.2 \%$ y sólo $1.9 \%$ solicitó un crédito mayor a $\$ 20,000$. Destaca que para la mitad de las mujeres $(50.5 \%)$ la cantidad del crédito recibido no fue suficiente. Al momento de entrevistarlas, $66 \%$ reportó que aún tenía su negocio, mientras que $34 \%$ expresó que le fue imposible mantenerlo. Al respecto, los testimonios siguientes expresan que MES está pensado para personas pobres que destinan el ingreso a la sobrevivencia y no está diseñado para establecer microempresas que representen una fuente de empleo y autoempleo constante:

"El dinero ha servido para que tengamos vida, para que tengamos comida” (Entrevista Jazmín, 2014).

"No me sirvió mucho, fue poco dinero, lo usé para comprar una cortadora industrial para mi taller, pero no me alcanzó" (Entrevista Adriana, 2014).

Las microempresas instaladas tienen los siguientes giros: $44.4 \%$ corresponde a venta de abarrotes, verduras, frutas, mariscos, carne y pollo; $19.4 \%$ a ventas por catálogo; $17.6 \%$ a elaboración y venta de alimentos; $11.3 \%$ a servicios varios: estéticas, sastrerías, refaccionarias, viveros, venta de medicamentos, cibercafés y tortillerías; $6.3 \%$ a la elaboración y venta de artesanías y sólo $1 \%$ a ganadería y agricultura. Lo expresado por las y los funcionarios entrevistados pone de manifiesto que no había especial atención sobre el giro de los negocios apoyados:

"Al principio se financiaron proyectos de ganadería y agrícolas porque heredamos grupos con esos apoyos" (Entrevista Alberto, 2014).

"Los negocios tienen relación con los roles tradicionales de género, cocinas económicas, ventas de pozol, tamal, fritangas, toda clase de catá- 
logos; se financió una talabartería, una herrería pero no eran operadas por mujeres, sino negocios de los esposos" (Entrevista Elena, 2014).

"No hay regla, igual les dimos el crédito a la que vende pozol o la de ventas por catálogo" (Entrevista Elisa, 2014).

En cuanto al ámbito de influencia o el alcance que tienen los negocios desarrollados, destaca que seis de cada $10(61.4 \%)$ vende sus productos en el barrio o la colonia donde vive, lo que implica que seguramente sus clientes son familiares o vecinos, $25.3 \%$ los vende en el mercado local y localidades cercanas y $13.3 \%$ en otros municipios, en la capital del estado y fuera de Chiapas. Acerca de las condiciones físicas desde donde opera el negocio, cabe señalar que más de la mitad de las mujeres lo desarrolla en su casa (56\%), 29.3\% vende en la calle o de puerta en puerta y sólo $14.7 \%$ cuenta con un local formal.

El tipo de negocios que establecen las mujeres son sencillos y ofrecen productos poco elaborados, lo que fomenta que éstos generen ingresos sólo para la sobrevivencia básica y que desarrollen diversas actividades y oficios. Sobre los ingresos, en general son mínimos, lo que explica que fundamentalmente contribuyen a la sobrevivencia diaria y muy pocos generan activos para el negocio y excedentes para la familia. Así, ocho de cada 10 mujeres (77.3\%) declara recibir ingresos de su negocio y, de éstas, $72 \%$ reporta recibir un ingreso mensual menor a $\$ 2,000$, es decir apenas cubren el ingreso mensual de la línea de bienestar por persona en zona rural establecida por el Coneval en $\$ 1,614.65$ para 2014. El siguiente testimonio muestra el tipo de negocio y el rango de ingreso:

"Hago pan, tamales, taquitos fritos, vendo tortilla, hago pozol, la cocina es mi defensa. Cuando me va bien me quedan $\$ 150$ de ganancia" (Entrevista Melina, 2014).

Acerca del destino del ingreso que proviene de los negocios establecidos con el préstamo MES, 65.3\% declaró utilizarlos para la compra de alimentos para la familia y, en menor proporción, a cubrir eventualidades como enfermedades, defunciones, viajes, graduaciones y celebraciones familiares $(21.3 \%)$, pagar deudas $(8.0 \%)$ y, en números absolutos, sólo dos mujeres los usan para construir o pagar colegiaturas, comprar algo para sí mismas (ropa, zapatos o cosméticos) o artículos para el hogar (muebles y electrodomésticos). Estos datos muestran que estas mujeres se encuentran en situación de pobreza y que los ingresos que perciben por medio del negocio se usan para la subsistencia familiar.

Para finalizar esta sección, tenemos que las microempresas financiadas por MES se caracterizan por ser negocios sencillos, las mujeres realizan actividades poco simplificadas con mínima transformación, los ingresos que perciben se destinan en su mayoría a satisfacer las necesidades familiares inmediatas, las condiciones físicas de los negocios expresan que 
están instalados en su propia casa y otras recurren a la venta de productos de puerta en puerta, y el alcance que tienen se circunscribe al barrio o colonia donde viven, por lo que consideramos que se trata de microempresas de subsistencia. ${ }^{5}$

\section{Conclusiones}

La revisión de literatura especializada nos permitió identificar aspectos en los que nuestro estudio coincide con los resultados reportados por diversas investigaciones. Sobre el impacto de los programas de microcrédito en la pobreza femenina estamos de acuerdo con García-Pinar (2012), Zapata-Martelo et al., (2003) y Tunón-Pablos et al., (2007), ya que encontramos que la mayoría de las beneficiarias reciben ingresos muy bajos, que algunas de ellas valoran como positivos y que tienen un efecto favorable en sus vidas. Estos datos a partir del enfoque propuesto por VegaEstrada (2009, 2012 y 2014) nos permite observar que MES incide en las mujeres pobres (sujeto) más no en la pobreza (contexto) ni en el empobrecimiento (proceso).

En torno a la relación ingreso-consumo encontramos que, en general, se cumplen los hallazgos reportados por otros autores (García-Horta et al., 2014; Hidalgo-Celarié et al., 2005; García-Pinar, 2012; Millán-Valenzuela, 2009; Tunón-Pablos et al., 2007; Vázquez-Jiménez, 2012; Sampayo-Paredes y Hernández-Romero, 2009) en el sentido de que el microcrédito ayuda a mejorar la capacidad de gasto de las usuarias y a incrementar el nivel de consumo de los hogares pobres.

Respecto al destino de los ingresos derivados de los micronegocios concordamos con Cardero (2008) y Zapata-Martelo et al. (2004c) ya que nuestros resultados muestran que la mayoría del grupo estudiado de beneficiarias del programa MES está desarrollando pequeños negocios que les proporcionan la generación de un ingreso adicional y una alternativa de empleo a ellas o sus esposos, así como que el principal destino de este

${ }^{5}$ Díaz-Arreguín (2011) propone una clasificación de microempresas en tres categorías que nos parece útil: 1) microempresas de subsistencia, que son pequeńos negocios con un empleado que realiza todas las funciones, con muy pocas posibilidades de subsistir, escasa o ninguna maquinaria ni activos fijos, que realizan actividades que no requieren trasformación sustancial de materiales, utilidades mínimas, poco capital de inversión, tienden a destinar los ingresos del negocio y los pocos ahorros que tengan a la satisfacción de necesidades familiares, el empresario carece de visión emprendedora, escasa formalidad y sin registro contable y fiscal; 2) microempresas en proceso de consolidación, que cuentan con uno o hasta tres empleados, capacidad limitada para generar recursos que tienden a reinvertirse, escasa tecnología, personal capacitado y tendencias a incorporarse a una cadena productiva, predomina la mano de obra familiar aunque sin remuneración, con registro fiscal y de contabilidad; y 3) microempresas competitivas, que cuentan con capital humano preparado, fondos disponibles, redes empresariales, un mayor número de empleados que tienen conocimiento técnico, el microempresario tiene visión de largo plazo, mayor organización, cuentan con figura y registros fiscales, así como acceso a tecnología. 
ingreso se dirige a la alimentación de los hogares (65.3\%) y un porcentaje menor $(11.9 \%)$ a cubrir eventualidades o gastos inesperados.

Sobre el tema de si el solo acceso a recursos monetarios produce mejoría o bienestar en las mujeres y sus familias (Varela-Zúñiga, 2007) y si contar con financiamiento ha contribuido a dotar de cierta libertad económica a las mujeres (Hidalgo-Celarié et al., 2005), encontramos que el tema es más complejo ya que, si bien en algunos casos el crédito ha permitido que las mujeres instalen sus propias empresas y esto les otorga cierta libertad económica, en otros son los maridos o algún familiar cercano del sexo masculino quien se beneficia y usa el crédito para instalar sus propios negocios.

Respecto a los tipos de negocios, nuestro estudio coincide con los de García-Horta et al., (2014) y Zapata-Martelo et al., (2004c) al reportar que la gran mayoría de las mujeres se dedican a la venta al menudeo de productos perecederos y no perecederos, ventas por catálogo a través del autoempleo y elaboración y comercialización de alimentos, mientras que un porcentaje reducido se dedica a actividades de tipo microempresarial en talleres o artesanía y, en menor medida aún, a actividades agropecuarias.

Aportamos también el hecho de que poco más de la mitad (55\%) establece el negocio en su casa, lo que nos lleva a reflexionar si esto les permite o no una clara delimitación entre el trabajo de reproducción familiar no remunerado y el negocio.

Asimismo, nuestros hallazgos sobre las microempresas concuerdan con lo planteado por Díaz-Arreguín (2011) al expresar que éstas tienen características que las hacen diferenciarse aún dentro del propio sector microempresarial e identificamos que MES apoya mayoritariamente a microempresas de subsistencia (82.5\%) y, en menor medida, a microempresas en proceso de consolidación (14.4\%) y menos aún a las que clasifican como competitivas (3.1\%).

Sobre los aciertos de MES encontramos que el programa atiende a un sector de la población tradicionalmente desatendido por el Estado (mujeres pobres), asignándoles pequeños préstamos para instalar microempresas sociales. La perspectiva de Vega-Estrada (2014) nos permite identificar que MES representa un programa de atención al pobre al ofrecer una alternativa de empleo o autoempleo que únicamente se centra en la atención de las pobres (sujeto).

El hecho de que los ingresos obtenidos por medio de las microempresas se destinan mayoritariamente a atender las carencias de alimento diario de las mujeres y sus familias, también concuerdan con el planteamiento de este autor al señalar que se atiende una necesidad inmediata pero no se resuelve la situación de pobreza y que, por tipo de microem- 
presas que el programa fomenta, no es posible revertir el proceso de empobrecimiento.

En este artículo hemos presentado evidencias que muestran algunas inconsistencias del programa MES. Por ejemplo, que tiene una naturaleza contradictoria ya que en la documentación institucional se expresa que es un programa de microfinanciamiento, pero carece de la identificación de la focalización de la población, lo que es una característica fundamental en ese tipo de programas (Conde-Bonfil, 2005, 2007 y García-Horta, et al, 2014). Por otro lado, la forma de operar el programa obedece más a un programa de fomento económico, lo que se refrenda en el uso de la terminología para nombrar a las mujeres a las que se les asignan microcréditos. Así, en MES se les denomina "acreditadas o beneficiarias", términos inspirados en los programas sociales que proporcionan un beneficio a la población asignándole un rol pasivo de receptor de algo, mientras que en los programas de microfinanciamiento se emplea el concepto "usuarias" que pueden ser personas físicas o jurídicas y que, en la definición, contempla un rol más activo de la persona que tiene derechos y compromisos respecto al uso del microcrédito que se le otorga en préstamo. Cabe señalar que en toda la literatura especializada que consultamos no existe esta distinción de los términos y que en todos los estudios se usa el concepto "beneficiaria". Al respecto consideramos que resulta pertinente distinguir uno de otro si mediante los programas de microfinanciamiento se pretende contribuir al empoderamiento femenino.

Otro rasgo característico de los programas de microfinanciamiento es el diseño de acciones de seguimiento, acompañamiento y capacitación atendiendo las necesidades específicas de las microempresas (MungarayLagarda, et. al., 2013). En el caso de MES observamos que no existe un esquema de capacitación consistente para sus microempresarias y que ha venido de más a menos a lo largo de los tres periodos identificados. También encontramos una desarticulación entre el objetivo del programa, los giros impulsados y la capacitación impartida.

En cuanto a las microempresas, encontramos otra debilidad institucional ya que MES fomenta la instalación y creación de empresas, pero no establece que cuenten con figura legal, lo que las destina a operan en la informalidad y les niega la posibilidad de recibir otros apoyos, que podrían ayudarles a incrementar su capital productivo. Es decir, MES promueve la instalación de microempresas de subsistencia, sin capacitación adecuada y figuras legales apropiadas y, con esto, les niega la posibilidad de encaminarse a una etapa de consolidación. Otra cuestión que consideramos que se debe distinguir en MES, si se pretendiera fomentar empleo y autoempleo permanente y sustentable, es el uso de los conceptos instalación y ampliación de empresas ya que no son lo mismo, refieren a dos 
fases distintas de las microempresas y a cada una de ellas le correspondería un esquema de acompañamiento y capacitación adecuado.

Finalmente, con nuestro estudio aportamos que se ha sobrevalorado el potencial del microcrédito como un programa de gobierno para combatir la pobreza femenina, ya que la evidencia que presentamos sobre el caso de MES muestra que esta iniciativa está impulsando la creación de microempresas de subsistencia que no constituyen una opción de empleo o autoempleo que incida en revertir el proceso de empobrecimiento de las mujeres.

\section{Fuentes consultadas}

Alpízar, Carlos A. y Claudio González-Vega (2006), "El sector de las microfinanzas en México”, en Carlos A. Alpízar y Claudio González-Vega (aut.), Los mercados de las finanzas populares y rurales en México. Una visión global rápida sobre su utilidad y alcance, USAID, Ciudad de México, México.

Apodaca, Pedro Miguel (1999), "Evaluación de los resultados y del impacto", Revista de Investigación Educativa, 17 (2), Ediciones de la Universidad de Murcia, Murcia, España, pp. 363-377, <http:// revistas.um.es/rie/article/view/121921>, 22 de marzo de 2015.

Arellano-Gault, David y Víctor G. Carreón-Rodríguez, Gustavo A. del Ángel-Mobarak, Fausto Hernández-Trillo, Alfredo Ramírez (2006), "Evaluación de resultados del Programa Nacional de Financiamiento al Microempresario, correspondiente al ejercicio fiscal 2006", Centro de Investigaciones y Docencia Económicas, A. C., Ciudad de México, México, <http://www.economia.gob. $\mathrm{mx} /$ files/transparencia/pronafim_eval_2006.pdf>, 20 de octubre de 2013.

Bustos-Villegas, José Luis (2010), “Microcréditos y vulnerabilidad: una evaluación de impacto para el caso mexicano", en La economía del campo mexicano: tendencias y restos para el subdesarrollo, Antonio Yúñez Naude, Fabiola Rivera Ramírez, María de los Ángeles Chávez Alvarado, José Jorge Nava Rivera y J. Edwards Taylor, El Colegio de México, Ciudad de México, México, <https://es. scribd.com/document/236456726/2-Microcredito $>, 28$ abril de 2017. 
Cardero, María Elena (2008), "Microfinanciamiento y mujeres pobres", Perfiles Latinoamericanos, 16 (32), Facultad Latinoamericana de Ciencias Sociales México, Ciudad de México, México, pp. 151$182<$ http://perfilesla.flacso.edu.mx/index.php/perfilesla/article/ view/179>, 16 de junio de 2015.

Conde-Bonfil, Carola (2007), "Contribución de las microfinanzas en la lucha contra la pobreza, en pro de la equidad de género y en la construcción de capital social”, 1st International CIRIEC Research Conference on the Social Economy, 22-25 de octubre, Victoria, Canadá, <http://www.academia.edu/728626/ Contribuci\%C3\%B3n_de_las_microfinanzas_en_la_lucha_contra_la_pobreza_en_pro_de_la_equidad_de_g\%C3\%A9nero_y_ en_la_construcci\%C3\%B3n_de_capital_social>, 14 de abril de 2014.

Conde-Bonfil, Carola (2005), "Orientación de los servicios microfinancieros hacia los más pobres”, Economía, Sociedad y Territorio, V (17), El Colegio Mexiquense, A. C., Toluca, Estado de México, México, pp. 161-218, <http://est.cmq.edu.mx/index.php/est/ article/view/320/780>, 19 de mayo de 2015.

Coneval (Consejo Nacional de Evaluación de la Política de Desarrollo Social) (2012), Pobreza y género en México. Hacia un sistema de indicadores, Coneval, Ciudad de México, México.

Coneval (Consejo Nacional de Evaluación de la Política de Desarrollo Social) (2009), Diagnóstico de las políticas públicas de microcrédito del gobierno federal, México, El Colegio de México, Ciudad de México, México.

Cotler-Ávalos, Pablo y Eduardo Rodríguez-Oreggia (2010), "Microfinanzas y la tenencia de activos no financieros en México", Investigación Económica, 69 (274), Ciudad de México, México, pp. 63-86, $<$ http://www.scielo.org.mx/scielo.php?script=sci_arttext\&pid=S $018516672010000400004 \& \operatorname{lng}=e s \&$ tlng=es $>, 10$ de enero de 2014.

Delalande, Laure y Christophe Paquette (2007), "El impacto de las microfinanzas en la reducción de la vulnerabilidad. Caso de un microbanco rural en México", Trace. Travaux et Recherches dans les Amériques du Centre, Centro de Estudios Mexicanos y Cen- 
troamericanos, núm. 52, Ciudad de México, México, pp. 63-77 < https://trace.revues.org/588>, 8 de septiembre de 2014.

Díaz-Arreguín, Sandra (2011), "La microempresa en el desarrollo regional de México", Memorias, 10 (17), Universidad Cooperativa de Colombia, Bogotá, Colombia, pp. 21-32<http://revistas.ucc.edu. co/index.php/me/article/view/121.Colombia>, 12 de febrero de 2016.

Esquivel-Martínez, Edgar Horacio (2010), “Medición del efecto de las microfinanzas en México", Comercio Exterior, 60 (1), Bancomext, Ciudad de México, México, pp. 9-27, < http://revistas.bancomext. gob.mx/rce/magazines/134/1/09_ESQUIVEL_microfinanzas. pdf>, 17 de octubre de 2015.

García-Horta, José Luis, Emma Zapata-Martelo, Esteban Valtierra-Pacheco y Laura Garza-Bueno (2014), "El microcrédito como estrategia para atenuar la pobreza de las mujeres, ¿cuál pobreza?”, Estudios Fronterizos, 15 (30), Universidad Autónoma de Baja California, Tijuana, México, pp. 97-126 <http://www.scielo.org. $\mathrm{mx} / \mathrm{scielo}$.ph p ? s cript $=\mathrm{sci}_{-}$art text $\&$ pid $=$ S018769612014000200004>, 13 de enero de 2015 .

García-Pinar, Raquel (2012), “Microcréditos, pobreza y género: consideraciones generales para la elaboración de programas de desarrollo vinculados a las microfinanzas con enfoque de género", Revista de Microfinanzas y Banca Social, núm. 1, Publicaciones Cajamar, Almería, España, pp. 91-114, <http://www.publicacionescajamar. es/publicaciones-periodicas/revista-de-microfinanzas-y-bancasocial-mbs/1/576/>, 7 de enero de 2014.

González-Martínez Roxana y M. Basaldúa-Hernández (2009), El impacto que tienen los programas gubernamentales de financiamiento en las microempresas de Querétaro, Memorias del programa verano de la ciencia 2010, Universidad Autónoma de Querétaro, Querétaro, México, <http:/www.uaq.mx/investigacion/difusion/ veranos/memorias-2010/12\%20Verano\%20Ciencia\%20Region\%20Centro/UAQ\%20Gonzalez\%20Martinez.pdf>, 25 de octubre de 2013.

Hidalgo-Celarié, Nidia, Reyes Altamirano-Cárdenas, Emma ZapataMartelo y Beatriz Martínez-Corona (2005), "Impacto económi- 
co de las microfinanzas dirigidas a mujeres en el estado de Veracruz, México", Agrociencia, 39 (3), Colegio de Postgraduados, Montecillo, México, pp. 351-359 <http://www.redalyc.org/ pdf/302/30239312.pdf>, 15 de agosto de 2014 .

López-Lira, Nidia (2007) "Elementos de integración de empresas comerciales en el oriente del Estado de México en los primeros años del siglo XXI", Contaduría y Administración, núm. 221, enero-abril, Universidad Nacional Autónoma de México, Ciudad de México, México, pp. 109-136, <http://www.redalyc.org/pdf/395/39522106. pdf>, 11 de febrero de 2016.

Medina-Núńez, Ignacio y Ángel Florido-Alejo (2010), "Microcrédito y desarrollo: financiamiento de proyectos sociales”, Revista Espiral, XVI (47), Universidad de Guadalajara, Guadalajara, México, pp. 105-137, <http://www.redalyc.org/articulo.oa?id=13811910004>, 22 de noviembre de 2013.

Mejía-Flores, Rocío (2004), "Instituciones y programas para los desbancarizados en el Distrito Federal", Comercio Exterior, (54) 7, Bancomext, Ciudad de México, México, pp. 584-597, <http:// revistas.bancomext.gob.mx/rce/magazines/68/4/RCE4.pdf>, 11 de enero de 2016.

Millán-Valenzuela, Henio (2009), Pobreza y microfinanciamiento: una evaluación de impacto, El Colegio Mexiquense, A. C., Toluca, México.

Mungaray-Lagarda, Alejandro, Michelle Texis, Martín Ramírez-Urquidi, José G. Aguilar y Natanael Ramírez (2013), "Emprendimiento solidario y desarrollo de microempresas de base social: una experiencia de trabajo conjunto y aprendizaje colectivo en Baja California", 4th International CIRIEC, Research Conference on the Social Economy, 24-26 de octubre, Amberes, Bélgica, <http:// www.ciriec-conference.org/images/upload/pdf/PAPERS/128_ texis_txt.pdf>, 16 de febrero de 2016.

Mungaray-Lagarda, Alejandro y Martin Ramírez-Urquidy (2007), “Capital humano y productividad en microempresas", Investigación Económica, LXVI (260), Facultad de Economía, UNAM, Ciudad de México, México, pp. 81-115<http://www.redalyc.org/articulo.oa?id=60126003> , 23 de enero de 2016. 
Pomar-Fernández, Silvia, Araceli Rendón-Trejo y Raúl Hernández-Mar (2006), "La microempresa. Reconociendo su importancia", X Congreso anual de la Academia de Ciencias Administrativas A. C., 2-5 de mayo de 2006, San Luis Potosí México, <http://acacia. org.mx/busqueda/pdf/P14T17-1.pdf>, 18 de febrero de 2015.

Rossi, Peter Henry, Howard E. Freeman y Mark W. Lipsey (2004) (1999), Evaluation. A systematic Approach, Sage, Los Ángeles, Estados Unidos de América.

Sampayo-Paredes, Humberto y Oliverio Hernández-Romero (2009), "Las instituciones microfinancieras. En las condiciones de vida, ingreso y cultura financiera y empresarial. El caso Finsol TexcocoChimalhuacán, México", Argumentos, 21 (56), Universidad Autónoma de México, unidad Xochimico, Ciudad de México, México, pp. 141-167 <http://www.scielo.org.mx/scielo. php?script=sci_arttext\&pid=S0187-57952008000100008>, 12 de julio de 2014.

Sedem (Secretaría para el Desarrollo y el Empoderamiento de las Mujeres) (2014), "Segundo Informe. Sedem", Gobierno del Estado de Chiapas, Tuxtla Gutiérrez, México.

Sedem (Secretaría para el Desarrollo y el Empoderamiento de las Mujeres) (2011a), "Reglas de operación. Fideicomiso Público de Inversión, administración y fuente alterna de pago: Fondo de Apoyo a la Economía Social (FAES)", Gobierno del Estado de Chiapas, Tuxtla Gutiérrez, México.

Sedem (Secretaría para el Desarrollo y el Empoderamiento de las Mujeres) (2011b), "Una semilla para crecer cumple 10 años", Mujeres Poder, núm. 2, Gobierno del Estado de Chiapas, Tuxtla Gutiérrez, México, pp. 3-5.

Tuñón-Pablos, Esperanza, Rolando Tinoco-Ojanguren y Armando Hernández-de la Cruz (2007), "Género y microfinanciación: evaluación de un programa de micro créditos para mujeres en el Estado de Tabasco", La Ventana, núm. 26, Universidad de Guadalajara, Guadalajara, México, pp. 41-69.

Varela-Zúñiga, María del Rosario (2007), “La promoción del desarrollo local a través de los programas de microcrédito para las mujeres 
en la ciudad de Torreón, Coahuila: una evaluación desde la perspectiva de género", en Rocío Rosales-Ortega (coord.), Desarrollo local: teorías y prácticas socio territoriales, Porrúa-Universidad Autónoma Metropolitana, unidad Iztapalapa, Ciudad de México, México, pp. 171-198.

Vázquez-Jiménez, Imelda Lorena (2012), Evaluación de los resultados del programa de microcréditos y de capacitación en los prestatarios de tres microfinancieras en Ciudad Obregón Sonora, México, Instituto Tecnológico de Sonora, Ciudad Obregón, México, <http://www. itson.mx/publicaciones/Documents/tesisdoct/evaluaciondelosresultadosdelprograma.pdf>, 14 de abril de 2014 .

Vega-Estrada, Sergio de la (2014), Para contender con la pobreza, Universidad Autónoma Metropolitana, Unidad Xochimilco-Miguel Ángel Porrúa, Ciudad de México, México, pp. 138.

Vega-Estrada, Sergio de la (2012), "Tratamiento de la pobreza", Red pobreza, Ciudad de México, México, <http://www.redpobreza. org.mx/index.php/documentos/doc_view/5-tratamiento-de-lapobreza.html>, 23 de noviembre de 2015.

Vega-Estrada, Sergio de la (2009), "Visión UAM sobre pobreza. Foro 2009”, Veredas, 10 (19)Universidad Autónoma Metropolitana, unidad Xochimilco, Ciudad de México, México, pp. 153-168.

Zapata-Martelo, Emma, Laura Elena Garza-Bueno, Josefina López-Zavala, Miriam Hernández-Neri, Pedro Pablo Ramírez-Moreno, María Isabel Ramos-Ávila, Camelia Reyes-Emba, Margarita Illescas-Montes de Oca (2003), CAME. Una experiencia de finanzas populares en el oriente del área metropolitana de la Ciudad de México, CAME Finanzas Populares-Colegio de Posgraduados-Área de Género Mujer Rural-Plaza y Valdés Editores, Montecillo, México.

Zapata-Martelo, Emma, Laura Elena Garza-Bueno, Josefina López-Zavala, Pedro Pablo Ramírez-Moreno, María Isabel Ramos-Ávila, Miriam Hernández-Neri, Camelia Reyes-Emba (2004a), Voces desde CAME: El impacto de los microcréditos, CAME Finanzas Populares-Colegio de Posgraduados-Área de Género Mujer RuralPlaza y Valdés Editores, Montecillo, México. 
Zapata-Martelo, Emma, Josefina López-Zavala, Miriam HernándezNeri, Laura Elena Garza-Bueno, Pedro Pablo Ramírez-Moreno, María Isabel Ramos-Ávila, Camelia Reyes-Emba (2004b), Microfinanciamiento y empoderamiento de mujeres rurales, CAME Finanzas Populares-Colegio de Posgraduados-Área de Género Mujer Rural-Plaza y Valdés Editores, Montecillo, México.

Zapata-Martelo, Emma, Pablo Ramírez-Moreno, Laura Elena GarzaBueno, Josefina López-Zavala, Miriam Hernández-Neri, María Isabel Ramos-Ávila, Camelia Reyes-Emba (2004c) Microfinanciamiento y pobreza, CAME Finanzas Populares-Colegio de Posgraduados-Área de Género Mujer Rural-Plaza y Valdés Editores, Montecillo, México.

\section{Entrevistas:}

Adriana, 36 años, Tuxtla, 2014.

Alberto, subsecretario de Programas Sociales de Banmujer (2000-2004), Tuxtla, 2014, primer periodo.

Cinthia, delegada de la Región Altos, (2001-2006), San Cristóbal, 2016, primer periodo.

Elena, delegada de la región I Centro (2001-2005) y Directora de Capacitación y proyectos productivos (2005-2011), Tuxtla, 2014, primer y segundo periodo.

Elisa, enlace municipal de la Sedem en Ocozocoautla (2013-2014), Ocozocoautla, 2014, tercer periodo.

Jazmín, 64 años, ejido Dr. Domingo Chanona, 2014.

Melina, 37 años, localidad Barranca Honda, 2014, ejercicio fiscal 2013.

Rocío, jefa del Departamento de programas de microfinanciamiento de la Subsecretaria de Economía Social-Banmujer de la Sedem, (2003-2014), Tuxtla, 2013, Tres periodos. 
Sofia, jefa del Departamento de Formación y Capacitación de la Dirección de Servicios No Financieros de Banmujer (2000-2009), Tuxtla, 2014, primer periodo e inicio del segundo.

Recibido: 6 de mayo de 2016. Corregido: 2 de septiembre de 2016. Aceptado: 19 de abril de 2017.

Emma del Carmen Aguilar-Pinto. Doctora en Ecología y Desarrollo Sustentable por El Colegio de la Frontera Sur. Entre sus publicaciones destacan: en coautoría "Los gobiernos locales y la promoción del desarrollo", en Villasana Benítez y Trujillo Olivera (coords.), Avances y perspectivas interdisciplinarias para el Desarrollo, UNACH-Universidad Rey Juan Carlos, San Cristóbal de las Casas, México, pp. 144-156 (2011); "Liderazgo, intermediación económica y políticas estatales en una región cafetalera del sureste de México", Revista Concurrencias y Convergencias Latinoamericanas, 3 (2), Asociación Latinoamericana de Sociología, Brasil, pp. 179-188 (2010); "Desarrollo local y género. Mujeres rurales en Chiapas", en Juana Aznar Márquez, Ana Martí, María Jesús Navarro y Anastasia Téllez (coords.), Desarrollo y trabajo de las mujeres en el medio rural, Icaria, Barcelona, España, 117-136 (2009); "Cafeticultura indígena en Yajalón: un escenario al margen del Comercio justo", Revista Pueblos y Fronteras, 4 (7), PROIMMSE-UNAM, Ciudad de México, México, pp. 157-186 (2009).

Esperanza Tuńón-Pablos. Doctora en sociología por la Universidad Nacional Autónoma de México. Actualmente es investigadora del Departamento de Sociedad y Cultura en El Colegio de la Frontera Sur. Es miembro del Sistema Nacional de Investigadores, nivel III. Su línea de investigación actual es desigualdades de género y su articulación con condiciones de pobreza y salud. Entre sus últimas publicaciones destacan: en coautoría "Risk factors for overweight and obesity among Mexican children in New York", International Journal of Population Research, volumen 2016, Hindawi, doi: 10.1155/2016/2420167 (2016); “Obesidad: el peso de las desigualdades de género entre las y los indígenas chontales de Nacajuca, Tabasco", Género y salud en cifras, 12 (1), Secretaria de Salud, Ciudad de México, México, pp. 27-40 (2014); Género y migración, El Colegio de la Frontera Sur-El Colegio de la Frontera Norte-El Colegio de Michoacán-Centro de Investigaciones y Estudios Superiores en Antropología Social, San Cristóbal de las Casas, México (2012); Género y 
cultura institucional, El Colegio de la Frontera Sur-REGEN, San Cristóbal de las Casas, México (2011).

Federico Morales-Barragán. Doctor en Ecología y Desarrollo Sustentable por el Colegio de la Frontera Sur. Actualmente es investigador del Centro de Investigaciones Multidisciplinarias sobre Chiapas y la Frontera Sur de la Universidad Nacional Autónoma de México. Su línea de investigación actual es Teorías y políticas de desarrollo regional. Entre sus últimas publicaciones destacan: "Desafíos para el diseño de políticas en los sistemas urbano rurales: aportes del enfoque territorial", en Carlos Brambila (coord.), Prioridades de investigación sobre pobreza y desarrollo, Tecnológico de Monterrey, Ciudad de México, México, pp. 181-202 (2015); "Cooperación transfronteriza: una metodología para su estudio", en Willy Soto (ed.), Ciencias sociales y relaciones internacionales: nuevos enfoques desde América Latina, Universidad Nacional, Heredia, Costa Rica, pp. 159-180 (2015); "Construcción de políticas para corredores biológicos. ¿Hacia un comanejo adaptativo?”, en Rocío Rosales y Ludger Brenner (coords.), Geografía de la Gobernanza. Dinámicas multiescalares de los procesos económico-ambientales, Universidad Autónoma Metropolitana Unidad Iztapalapa-Siglo XXI, Ciudad de México, México, pp. 324-348 (2015). 